\title{
Applicability of ABR Service to Internet Applications
}

\author{
Mika Ishizuka \\ NTT Information Sharing Platform Laboratories \\ 3-9-11, Midori-cho, Musashino-shi, Tokyo, 180-8585 Japan \\ ishizuka.mika@lab.ntt.co.jp
}

\begin{abstract}
Many studies on the performance of the ABR service category have been done. Most of these were simulation studies, and most evaluated performance only in the ATM layer. However, simulations do not provide a complete picture of how ABR service is used by actual applications running on existing networks. Few studies of how applications operate in a real environment have been done. We have used a testbed ABR network to examine the applicability of Internet applications. In this network, we tested four types of application: an FTP application (bulk data transfer), and a WWW application (interactive flow), a Video on Demand (VOD) application that used Motion JPEG, and an IP telephony application. As a result, we found that ABR service can be applied to these applications and determined how to set the best ABR parameters to ensure the best performance of each application.
\end{abstract}

\section{Introduction}

Asynchronous Transfer Mode (ATM) is widely used to achieve high-speed communication. ATM has a number of service categories. Best effort service is suitable for Internet applications because these applications send unpredictable and burst traffic. A typical example of a best effort service category is Unspecified Bit Rate (UBR) service. However, UBR service uses no form of traffic control, so unless each application controls its traffic flow properly, application performance will be degraded by cell loss when a network is congested. To solve this problem, Available Bit Rate (ABR) service category was designated in the ATM Forum [1]. ABR service can avoid ATM layer congestion and minimize cell loss, because it uses a closed loop feedback rate control mechanism. In ABR service, a source end system calculates the Allowed Cell Rate (ACR) based on feedback information from a network. ABR service can also provide a Minimum Cell Rate (MCR) guarantee.

Many studies on the performance of the ABR service category have been done [2, 3]. Most of these were simulation studies, and most evaluated performance only in the ATM layer, (for example, ATM link utilization or the maximum queue length in ATM switches). However, simulations do not provide a complete picture of how ABR service is used by actual applications running on existing networks. Few studies of how applications operate in a real environment have been done.

We have used a testbed ABR network to examine the applicability of Internet applications. In this network, I tested four types of application. Two were TCP 
applications: an FTP application (bulk data transfer), and a WWW application (interactive flow). The other two were real-time applications: a Video on Demand (VOD) application that used Motion JPEG, and an IP telephony application.

The paper is organized as follows. In section 2, we explain ABR feedback control mechanism. In section 3, we depict configuration of our experiment. Then in section 4, we show experiment results and discuss theses results. Finally, in section 5, we conclude the paper.

\section{ABR Behavior}

In this section, we briefly describe ABR feedback control mechanism. In ABR service, a Resource Management cell (RM cell) is used to form closed loop feedback control system. A source end system (SES) is given information about congestion through RM cells. According to these information, an SES calculates allowed cell rate (ACR), and adjusts sending cell rate to ACR. An ATM switch indicates information about congestion in one of three ways: EFCI, Relative Rate, and Explicit Rate (ER) marking. In this experiment, ATM switches supporting only EFCI marking are used. Therefore we explain EFCI marking here. In [1, 4], the others are described in detail.

- An SES generates an RM cell every after Nrm-1 cells.

- An ATM switch sets the EFCI bit in a data cell when its queue length exceeds its threshold.

- A destination end system sends the RM cell back to the SES, when an RM cell reaches it. At this time, congestion indication bit in the RM cell is set when the EFCI bit in the last received data cell was set.

- When an SES receives an RM cell, the SES calculates ACR as follows:

- if the CI bit in the RM cell is set $\mathrm{ACR}:=\max (\mathrm{ACR}-\mathrm{RDF} * \mathrm{ACR}, \mathrm{MCR})$,

- else $\mathrm{ACR}:=\min (\mathrm{ACR}+\mathrm{RIF} * \mathrm{PCR}, \mathrm{PCR})$.

\section{Experiment Configuration}

\subsection{Network Configuration}

The network configuration we used is shown in Fig. 1. 


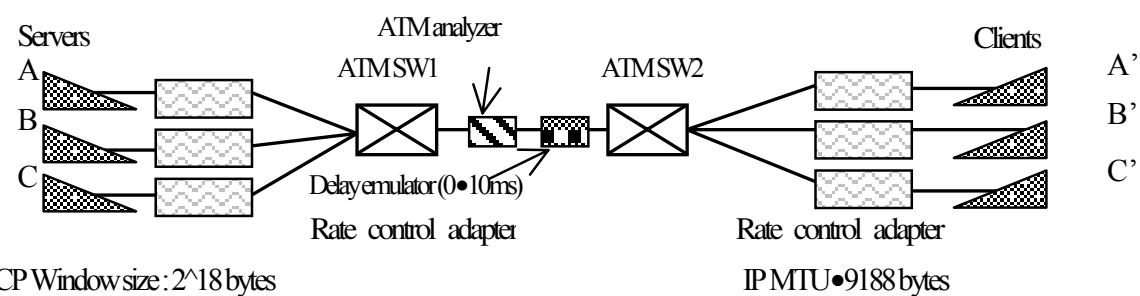

Bandwidth: $10 \mathrm{Mbps}$

Fig. 1. Network configuration

- In each ATM switch, cells transmitted to the same output port share buffer memory, which size is configurable. In this configuration, it was set to $4 \mathrm{k}$ cells. Threshold for EFCI marking was set to 127 cells.

- As for end systems, SS20+Solaris2.4 (except C), and Ultra2+Solaris 2.5.1(C) were used. Solaris 2.4 supports window scaling option [5] by applying consultingoption.

- Rate Control Adapters (RCA) work as ABR end systems. ABR parameters were set based on ATM Forum TM 4.0.

- PVCs were used to set up ATM connections.

- Delay was set by emulator. Default setting was $10 \mathrm{~ms}$.

\subsection{Applications Setting}

Traffic from A was sent to A', and that from B was to B'. These two connections offered background traffic using an FTP application. As for C to C', three applications were used, WWW, VoD, and IP Telephony.

In a WWW application, thirty identical WWW pages, which include a html (600bytes) and a gif file (300kbytes), were downloaded. Cache setting at a WWW client was disabled.

Table 1 shows other settings concerning to each application.

Table 1. applications used in this experiment.

\begin{tabular}{|l||l|l|}
\hline AP & protocol & parameters $\bullet$ CODEC, etc. \\
\hline \hline FTP & TCP & TCP maximum window size $2^{18}$ bytes \\
\hline WWW & TCP & TCP maximum window size 9148 bytes \\
\hline VoD & UDP & Motion JPEG $\bullet$ frame rate $=24 \mathrm{fps}$ \\
\hline IP Telephony & RTP(UDP) & Freephon[6] (with recovery techniques [7]). \\
\hline
\end{tabular}

\subsection{Performance Metrics}

- Common among three applications : cell rate between ATM SW1 and 2, and ACR of each connection. 
- FTP: throughput.

- WWW : response time. In this paper, interval between a request passing the ATM analyzer and the last cell of response data passing it is defined as response time.

- VOD : loss ratio that is indicated by the application.

- IP telephony : packet interval.

\section{Results and Analysis}

\subsection{WWW}

Three scenarios were tested to examine applicability of ABR to a WWW application: (1) A UBR connection for a WWW application without background traffic, (2) Three UBR connections sharing bandwidth, one of these connections for a WWW application and the others for an FTP application, (3) Three ABR connections sharing bandwidth, which use the same application as (2).

For each scenario, cell rate between ATM switches is shown in Figs. 2,3, and 4 (measurement interval is $100 \mathrm{~ms}$ ).

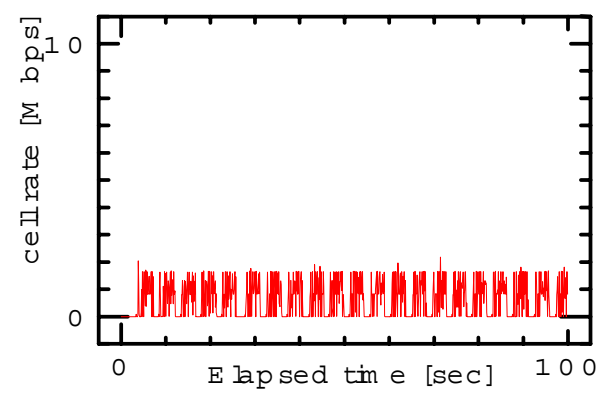

Fig. 2. Cell rate between switches for a UBR connection without background traffic

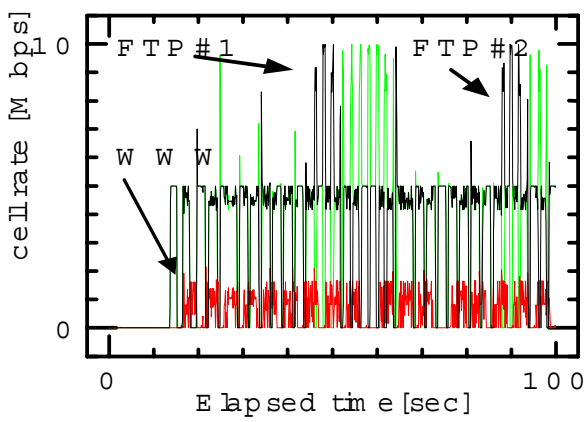

Fig. 3. Cell rate between switches for UBR connections with background traffic 


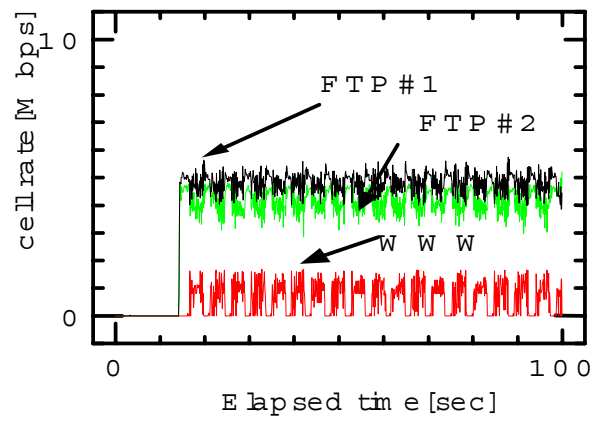

Fig. 4. Cell rate between switches for ABR connections with background traffic

From these figures, We found ABR is better than UBR, because the available bandwidth can be fairly assigned to each connection. Note that, when we use the ABR, a WWW application can be assigned a fair allocation of bandwidth even though its traffic is less than others. This is because the larger a VC shares bandwidth, the larger rate reduction the VC suffers.

Then, a response time and ftp throughput are shown in Tables 2, and 3. In Table 3, ABR parameters, RIF and RDF, are changed to determine how to set the best parameters for a WWW application. In both tables, response time is calculated about a html and a gif file respectively.

Table 2. WWW responce time and FTP throughput for a UBR connection.

\begin{tabular}{|c|c|c|}
\hline & No background traffic & UBR \\
\hline \hline Html response time[ms] & $2.9 \pm 0.1$ & $3.7 \pm 1.5$ \\
\hline Gif response time[s] & $3.22 \pm 0.15$ & $3.55 \pm 0.94$ \\
\hline ftp throughput[Mbps] & 8.9 & 7.7 \\
\hline
\end{tabular}

Table 3. WWW response time and FTP throughput for a ABR connection.

\begin{tabular}{|l|l||c|c|c|}
\hline RDF & & $1 / 16$ & $1 / 64$ & $1 / 256$ \\
\hline \hline \multirow{3}{*}{$1 / 16$} & Rtml[ms] & $4.1 \pm 0.8$ & $4.7 \pm 2.0$ & $4.4 \pm 1.5$ \\
\cline { 2 - 5 } & Gif[s] & $2.66 \pm 0.08$ & $2.68 \pm 0.10$ & $2.67 \pm 0.08$ \\
\cline { 2 - 5 } & ftp[Mbps] & 8.2 & 7.6 & 7.3 \\
\hline \multirow{3}{*}{$1 / 64$} & Html[ms] & $4.2 \pm 0.9$ & $4.2 \pm 0.8$ & $4.1 \pm 0.8$ \\
\cline { 2 - 5 } & Gif[s] & $2.65 \pm 0.07$ & $2.64 \pm 0.08$ & $2.63 \pm 0.06$ \\
\cline { 2 - 5 } & ftp[Mbps] & 9.0 & 8.8 & 8.7 \\
\hline \multirow{3}{*}{$1 / 256$} & Html[ms] & $4.3 \pm 0.9$ & $4.2 \pm 0.8$ & $4.2 \pm 0.8$ \\
\cline { 2 - 5 } & Gif[s] & $3.59 \pm 0.83$ & $2.67 \pm 0.09$ & $2.66 \pm 0.06$ \\
\cline { 2 - 5 } & ftp[Mbps] & 5.0 & 8.1 & 8.8 \\
\hline
\end{tabular}

: cell loss is observed.

As is shown in Table 3, for bulk data transfer such as an FTP application, $\mathrm{ABR}$ is the best service category because the available bandwidth can be fairly assigned to each connection and cell loss can be minimize. As a result, a TCP connection can fully utilize the available bandwidth by minimizing the overhead 
caused by TCP retransmission of lost data. The ABR parameters must be set to avoid both buffer starvation and buffer overflow; this means aggressive parameter setting is desirable for this kind of application.

As for a WWW application, when we use no flow control, that is, when we use a UBR, a WWW application cannot be assigned a fair allocation of bandwidth, so cell loss occurs for a WWW connection even though the WWW application uses less bandwidth than the background traffic. As a result, use of a UBR degraded the response time in presence of the background traffic. Furthermore, response time becomes unstable, due to data loss, when many segments are retransmitted. Actually, about $4 \%$ of data segment are retransmitted in case of UBR. These two problems can be solved by use of ABR service, that is, short and stable response time is enabled. To avoid TCP retransmission by using congestion control mechanism in ABR keeps the quality of interactive TCP applications high. ABR parameters must be set to keep the queue length short to reduce delay and to avoid cell loss. Therefore, contrary to the case of an FTP application, parameters setting for a WWW application should be conservative.

Considering above results, to ensure good performance for both applications, FTP and WWW, $(\mathrm{RIF}, \mathrm{RDF})=(1 / 64,1 / 64)$ is the best in this environment (number of connections is 3 , and ICR=PCR).

Finally, a response time and ftp throughput for three ICR settings are shown in Table 4. In addition, snapshot of ACR is shown in Fig. 5 ( $R D F=1 / 64, R I F=1 / 64$, $\mathrm{ICR}=\mathrm{PCR} / 10) \bullet A s$ is shown in Fig. 5, when ACR $>\mathrm{ICR}, \mathrm{ACR}$ is reset to ICR before next WWW page is transmitted $\bullet$ Therefore, a response time becomes longer by setting smaller ICR, though queue length can be kept low. From these results, small ICR setting is better, as long as a response time, when background traffic is not heavy, is not so worse.

Table 4. WWW response time and FTP throughput for an ABR connection when ICR is changed.

\begin{tabular}{|l|l||c|c|c|}
\hline ICR & RIF & $1 / 16$ & $1 / 64$ & $1 / 256$ \\
\hline \hline \multirow{3}{*}{ PCR } & Html[ms] & $4.2 \pm 0.89$ & $4.2 \pm 0.8$ & $4.1 \pm 0.8$ \\
\cline { 2 - 5 } & Gif[s] & $2.67 \pm 0.07$ & $2.65 \pm 0.08$ & $2.63 \pm 0.06$ \\
\cline { 2 - 5 } & ftp[Mbps] & 9.0 & 8.8 & 8.7 \\
\hline \multirow{3}{*}{ PCR/3 } & Html[ms] & $4.8 \pm 0.8$ & $4.6 \pm 0.2$ & $4.7 \pm 0.2$ \\
\hline \multirow{3}{*}{ PCR/10 } & Gif[s] & $3.21 \pm 0.13$ & $3.21 \pm 0.17$ & $3.21 \pm 0.23$ \\
\cline { 2 - 5 } & ftp[Mbps] & 9.0 & 8.8 & 8.7 \\
\hline & Html[ms] & $11 \pm 0.4$ & $11 \pm 0.3$ & $11 \pm 0.5$ \\
\cline { 2 - 5 } & Gif[s] & $3.23 \pm 0.19$ & $3.23 \pm 0.13$ & $3.20 \pm 0.16$ \\
\cline { 2 - 5 } & ftp[Mbps] & 9.0 & 8.8 & 8.7 \\
\hline
\end{tabular}

The following is the summary of this subsection. [Applicability of ABR service for TCP applications]

- For an FTP application, ABR is the best service.

- For an interactive application such as a WWW application, ABR service also works well, because short and stable response times are achieved. ABR parameters must be set to keep the queue length short to reduce delay and to avoid cell loss. 


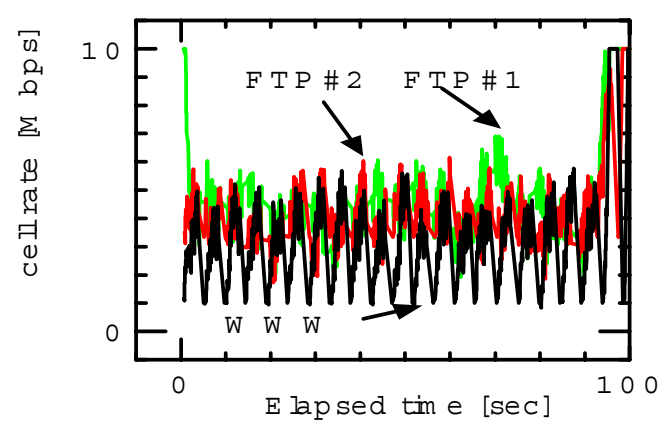

Fig. 5. Snapshot of $A C R$ when $R I F=R D F=1 / 64$, and $I C R=P C R / 10$

\subsection{VoD}

We made our measurements with a VoD application that uses UDP for data transmission. The CODEC of our VoD application is Motion JPEG. We consider three conditions for underlying networks: (1) A UBR connection for the VoD application without any other background load, (2) A UBR connections for the VoD application and two other UBR connections for background TCP traffic during 10 seconds, and (3) An ABR connections for the VoD application and two other ABR connections for background TCP traffic during 10 second. By observing video movies at the receiver side, we found the following results.

- In case of UBR connections with background traffic, we observe suspension of movie due to losses of movie frames. After the congestion period, the movie restart playing but lost part of the movie is skipped and is never displayed. As a result, we observe continuous loss of movie more than the half of the congestion duration.

- In case of ABR connections with background traffic, we observe delay in displaying movies during congestion periods. This is because ABR rate control adapter limits the transmission rate of video stream. After finishing the congestion period, the rate control adapter transmits the cells stored in its buffer at PCR and this results in high frame arrival rate at the receiver host. This causes overflow of the frame buffer at the receiver host and we observe approximately $10 \%$ loss of the movie at that period.

We show the transient cell rate characteristics in $100 \mathrm{~ms}$ time unit between the switches in Fig. 6 (UBR without background traffic), Fig. 7 (UBR with background traffic), and Fig. 8 (ABR with $\mathrm{RDF}=1 / 64$ and $\mathrm{RIF}=1 / 64$ ).

In Fig. 7, we can see that the share of VoD, which is UDP, is dominant and TCP traffic can obtain only a small share of the whole bandwidth. The total throughout for the two TCP connections was 0.7 Mbps. In case of Fig. 8, we used ABR connections and the total bandwidth is fairly shared by the three ABR connections. As a result, the total throughout for the two TCP connections was 6 Mbps.

As we mentioned above, when we use ABR, we observe loss in frames of VoD movie at the time when the background traffic terminated. To solve this traffic phenomenon, we propose two approaches. The first one is the reduction of video frame rate. The other one is the increase of MCR. 


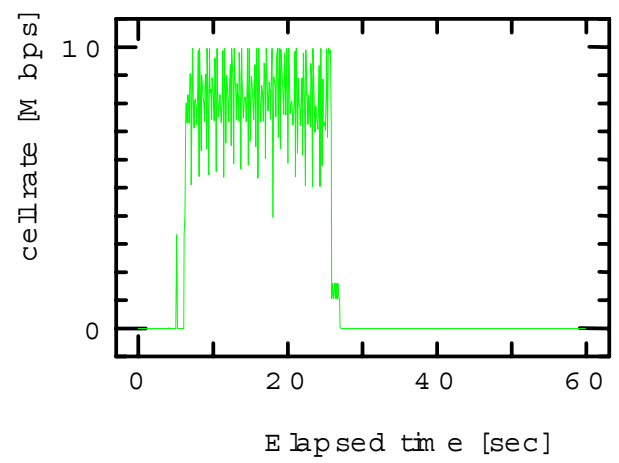

Fig. 6. Cell rate between switches for a UBR connection without background traffic.

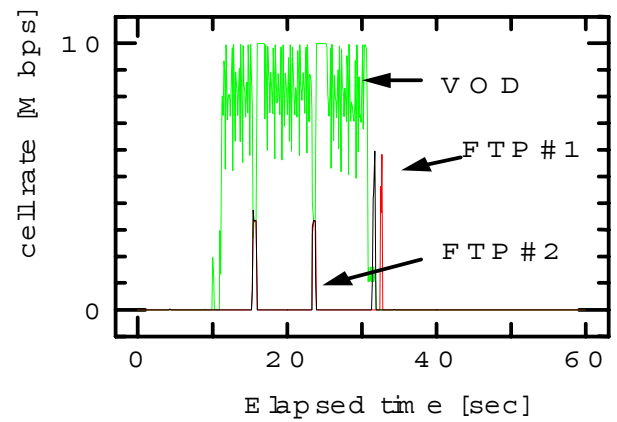

Fig. 7. Cell rate between switches for UBR connections with background

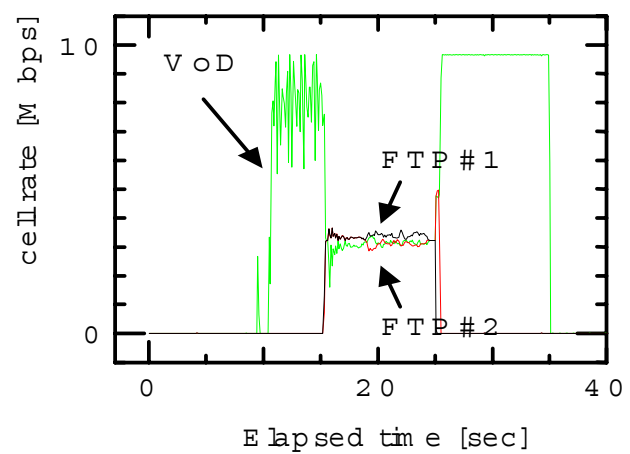

Fig. 8. Cell rate between switches for $A B R$ connection with $\mathrm{RDF}=1 / 64$ and $\mathrm{RIF}=1 / 64$

(1) Reduction of video frame rate

We reduce frame transmission rate from 24 fps by 1 fps steps and search the maximum frame rate at which the $\mathrm{VoD}$ application does not indicate any frame loss. We found $9 \mathrm{fps}$ is the maximum one. We show the results of the transient cell rate characteristics between the switches in Fig. 9 (UBR without background traffic), Fig. 10 (UBR with background traffic), and Fig. 11 (ABR). 


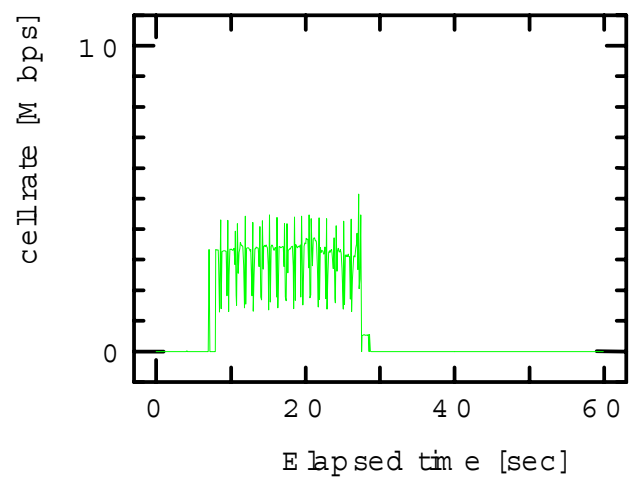

Fig. 9. Cell rate between switches for $9 \mathrm{fps}$ VoD and a UBR connection without background traffic

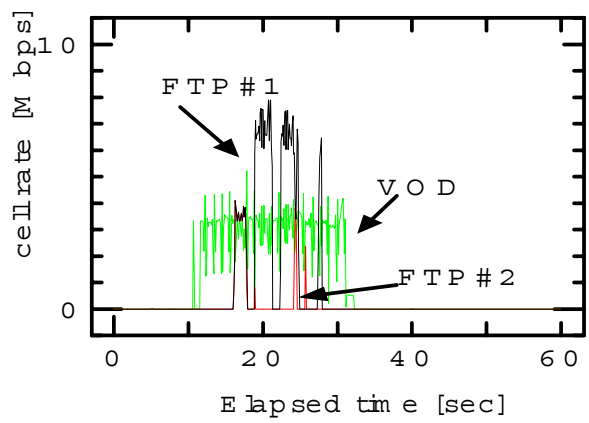

Fig. 10. Cell rate between switches for $9 \mathrm{fps}$ VoD and UBR connections with background traffic

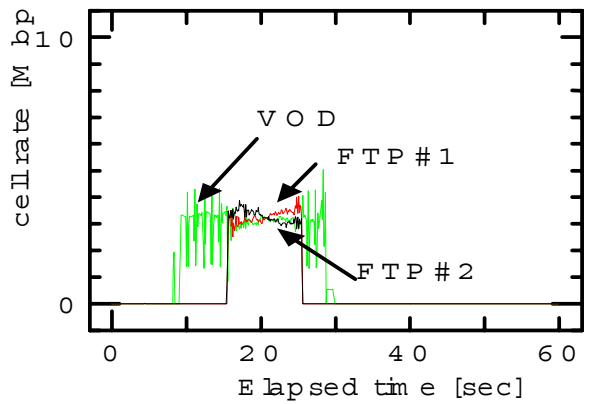

Fig. 11. Cell rate between switches for $9 \mathrm{fps} \operatorname{VoD}$ and $A B R$ connection with $\mathrm{RDF}=1 / 64$ and $\mathrm{RIF}=1 / 64$

In case of UBR (Fig. 10), we could not solve unfairness among UDP and TCP connections and the total throughput for two TCP connections is $2.0 \mathrm{Mbps}$. We also note that in this case, we observe suspension of movie at the receiver host and the 
VoD application indicates $10 \%$ of frame losses. In case of ABR with 9 fps for the video stream, although the video quality degrades due to the reduction of frame rate, we did not perceive any degradation in actual displayed movie comparing to the case for UBR without background traffic. We also note that we can maintain zero frame loss at application level for ABR with $9 \mathrm{fps}$. As we can see from the Fig. 11, by reducing the frame rate to $9 \mathrm{fps}$ that is $3 / 8$ of the original $24 \mathrm{fps}$, the corresponding cell rate is also approximately $3 / 8$ of the original cell

rate. This suggests a possibility to the dynamic rate adaptation for VoD. By investigating the maximum bit rate that does not cause frame loss for the original movies and dynamically controlling the frame rate proportional to (ACR/bit rate of movie), we shall maintain the quality of $\mathrm{VoD}$ even when the available bandwidth changes time to time.

(2) Increasing MCR

We investigate the effect of MCR by changing MCR from 0Mbps by 1 Mbps steps. The minimum MCR that does not cause loss in VOD frame is 7 Mbps. We show the cell rate between switches for this case in Fig. 12.

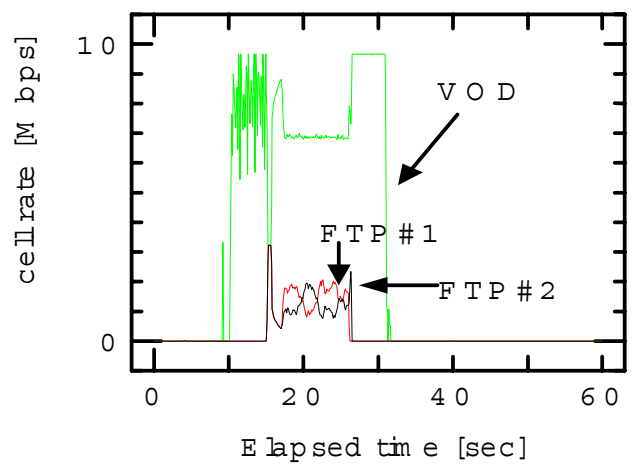

Fig. 12. Cell rate between switches for $A B R$ connections with $M C R=7 M b p s$ for $\mathrm{VoD}$

As we explained in section 4.1 with regard to WWW, the higher the transmission rate of a VC is, the larger rate reduction the VC suffers. Thus, the residual bandwidth is $3 \mathrm{Mbps}$ in our condition but the bandwidth share for VoD is less than that of the other VCs with MCR $=0$ Mbps. By observing the actual movies, we found $7 \mathrm{Mbps}$ is the smallest allowable MCR for VoD traffic to maintain smoothness of the video. This means that by allocating large MCR for VoD traffic, we can increase tolerance for the VoD traffic against background load. Here, the PCRs and the durations of the background connections specify the background load. We can find the maximum value of the parameters for background ABR connections to avoid a frame loss in VoD application as follows. 
( $\mathrm{R}$ - cell rate for the $\mathrm{VoD}$ connection during congestion) $* \mathrm{~T} /(\mathrm{PCR}-\mathrm{R})<$ receiver buffer size.

Here, $\mathrm{R}$ [Mbps] denotes the cell rate for the $\mathrm{VoD}$ connection when there is no background load, and $\mathrm{T}[\mathrm{s}]$ denotes the duration of congestion. We can approximate cell rate for the $\mathrm{VoD}$ connection during congestion as MCR.

In the above relationship, the receiver buffer size is unknown but we could estimate it from measurements.

The followings are the summary of the above results.

[Applicability of ABR service for $\mathrm{VoD}$ ]

(1) No unfairness occurs when we use ABR even if there coexists FTP traffic using TCP and VoD traffic using.

(2) We observed that the bandwidth used by the VoD is approximately $8.5 \mathrm{Mbps}$ so that it is larger than the fair share of the bandwidth. In that case, we can calculate allowed background load for it. By allocating MCR enough, we can enlarge the allowed background load.

(3) By using the currently available bandwidth information, such as ACR, we can maintain movie quality by dynamically updating transmission frame rate (namely, transmission cell rate). As we mentioned above, frame rate is approximately proportional to the cell rate. Thus we suggest the effectiveness of periodical frame rate updating method by using mean bit rate of a video and mean available bandwidth in a certain interval.

\subsection{IP Telephony}

When RIF and RDF are set to 1/64, cell rate between switches is shown in Fig. 13. For comparison, Fig. 14 shows cell rate between switches when no background traffic is offered. From these figures, we can confirm share of each application is fair.

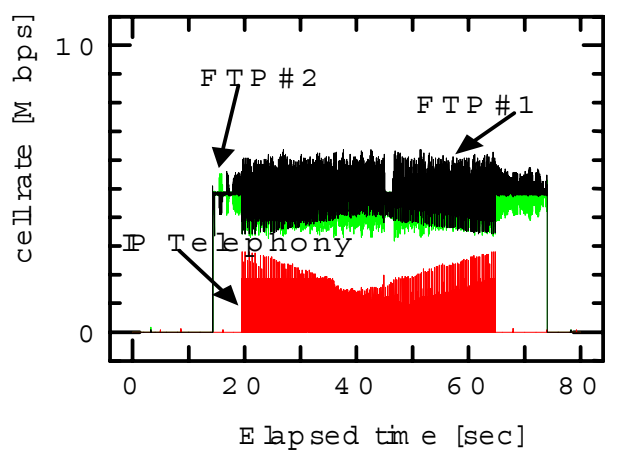

Fig. 13. Cell rate between switches for $A B R$ connections when $R I F=R D F=1 / 64$ 


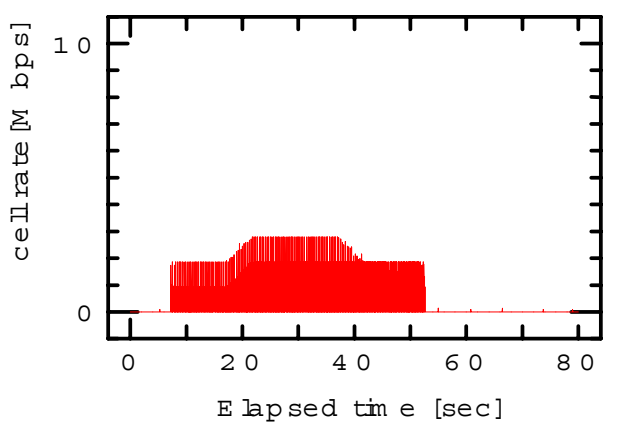

Fig. 14. Cell rate between switches for a UBR connection without background traffic

When we use a UBR, many silent periods occured. This is because the error recovery techniques in such an application do not work well when burst data is lost.

On the other hand, the ABR worked well in that we could hear undegraded sounds. This is because IP telephony uses relatively little bandwidth and has recovery techniques to be used in an Internet environment that does not guarantee any quality of service. The ABR parameters setting, as in the case of a WWW application, must keep the queue length short to reduce delay.

Delay variation, however, is caused by use of ABR, because cell rate is adjusted dynamically. Actually, packet interval for an IP Telephony without no background traffic differs from that using an ABR connection with background traffic at the order of milliseconds. Thus, only when a real-time application has some robustness for delay variation, ABR service is useful for the application. Furthermore, considering queueing delay in rate control adapter, the smaller bandwidth the application uses the better performance is achieved.

\section{Conclusion}

Overall, our findings show that the best ABR parameter setting should depend on applications. However, when users cannot set appropriate ABR parameters for each application, conservative setting to keep queue length low and to avoid buffer starvation is effective in most cases.

Even though ABR service is not primarily intended for real-time applications, the ABR can be effective for these applications when some conditions are satisfied. In addition, we have found that harmonization of ABR flow control and application level flow control increases the variety for which the ABR can be effectively used. 


\section{References}

1. ATM forum traffic management specification 4.0, The ATM Forum, (1996)

2. Ishizuka, M. et al.: Performance Analysis of TCP over ABR in High-Speed WAN Environment, IEICE Tarns. COMMUN, vol. E80-B, no.10, (1997)

3. Ohsaki, H. , Murata, M., Miyahara, H.: Parameter Tuning of Rate-Based Congestion Control Algorithm for ABR Service Class in ATM Networks, Int. Journal on Communication Systems, vol.16, no.7, (1998) 103-128

4. Saito, H., Kawashima, K., Kitazume, H., Koike, A., Ishizuka, M., Abe, A.: Performance Issues in Public ABR Service, IEEE Communication Magazien, vo.34, no.5, (1996) 4048

5. Jacabson, V., Braden, R., Borman, D.: TCP Extensions for High Performance, RFC1323, (1992)

6. http://www-sop.inria.fr/rodeo/fphone/

7. Bolot, J. C., Gare, A. V.: The case for FEC-based error +control for packet audio in the Internet, to appear in ACM Multimedia Systems 